\title{
A rede como estratégia metodológica da Política Nacional de Humanizaçãao: a experiência de um hospital universitário
}

Maria Lúcia Rodrigues Falk

Márcia Ziebell Ramos² Jennifer Braathen Salgueiro ${ }^{3}$

\section{Introdução}

A humanização das relações de trabalho e da assistência em saúde tem ocupado, nos últimos anos, espaços significativos de formulação de políticas públicas em diversos fóruns ligados à saúde no âmbito hospitalar. Esse tema aponta para a importância da ação e contextualização das dimensões do cuidar e do viver humano. O governo tem se empenhado na elaboração de políticas públicas capazes de oferecer maior ressonância e viabilidade na busca da qualidade e humanização hospitalar (Salgueiro et al., 2007). Possui, como premissas básicas: a valorização dos diferentes sujeitos implicados no processo de produção de saúde, bem como o fomento da autonomia e do protagonismo desses sujeitos; o aumento do grau de corresponsabilidade; o estabelecimento de vínculos solidários e de participação coletiva no processo de gestão; a identificação das necessidades sociais de saúde; a mudança nos modelos de atenção e gestão dos processos de trabalho, tendo como foco as necessidades dos cidadãos e a produção de saúde; o compromisso com a ambiência e a melhoria das condições de trabalho e do atendimento (Brasil, 2006).

Neste sentido a compreensão da saúde se dá como processo de produção, que, para além do binômio queixa conduta, considera a complexidade das relações entre os diferentes envolvidos nos processos de produção de saúde (trabalhadores, gestores e usuários). A humanização é então considerada "como processo de subjetivação, que se efetiva com a mudança nos modelos de atenção e de gestão em saúde, isto é, novos sujeitos implicados em novas práticas de saúde. Assim, pensar a saúde como experiência de criação de si e de modos de viver é tomar a vida em seu movimento de produção de normas, e não de assujeitamento a elas" (Benevides, Passos, 2005a, p.570).

O Ministério da Saúde propôs a criação da Política Nacional de Humanização (PNH), que traduz princípios e modos de operar no conjunto das relações entre todos que constituem o SUS. Sendo que, especialmente, o modo coletivo e cogestivo de produção de saúde e de sujeitos implicados nessa produção é que deve orientar a construção da PNH como política pública (Santos Filho, 2007). A proposta de humanizar deve estar fortemente vinculada com a reforma das modalidades clínicas, que se desenvolvem em hospitais, caso contrário corre o risco de ser banalizada e tratada com superficialidade.

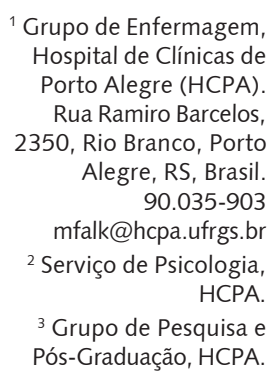
HCPA.

${ }^{3}$ Grupo de Pesquisa e Pós-Graduação, HCPA. 
Esse pensamento aposta no protagonismo do sujeito e na potencialidade do coletivo. Compreende o sujeito "como produto resultante de um funcionamento que é de produção inconclusa, é heterogenético, nunca havendo esgotamento total da energia potencial de criação das formas. É por isso que dizemos que a subjetividade é plural, polifônica, sem nenhuma instância dominante de determinação" (Benevides, 2005, p.23). O profissional da saúde, ao refletir sobre as condições e relações de trabalho e o seu modo de agir, pode inserir-se na realidade de uma maneira mais crítica e consciente (Backes, Lunardi Filho, Lunardi, 2006).

Assim, a política de humanização passa pela imbricação das linhas de ação da assistência, gestão e ensino, de modo a potencializar outros modos de fazer saúde em hospitais.

O presente artigo é um relato de experiência instituída no Hospital de Clínicas de Porto Alegre (HCPA), uma instituição pública e universitária ligada ao Ministério da Educação e à Universidade Federal do Rio Grande do Sul, que há 34 anos vem oferecendo atendimento de qualidade aos pacientes - na grande maioria, por intermédio do Sistema Único de Saúde (SUS) - e desenvolvendo atividades de ensino e pesquisa em saúde. Possui, como visão, ser um referencial público de alta confiabilidade em saúde. A proposta deste relato foi a de descrever a formação da rede de contatos como uma das estratégias criada pelo Grupo de Trabalho de Humanização (GTH) para implantação dos demais dispositivos da PNH no HCPA.

\section{Metodologia}

As redes representam um dispositivo preconizado pelo Ministério da Saúde para a implantação da Política Nacional de Humanização. Têm como objetivo a transversalização (Kamkhagi, 1986) nos processos de comunicação e modos menos "tradicionais" de se construírem as relações institucionais e de desdobramentos nos modos de fazer gestão.

Nesse sentido ativa-se "a produção de redes quentes, orgânicas ou redes sociais, que valorizam os serviços mais descentralizados e alteram as relações entre equipes localizadas nos espaços mais periféricos e aquelas equipes, serviços ou profissionais localizados em espaços mais valorizados e centrais" (Righi, 2006, p.87). Tais redes, dessa forma, constituem dispositivo, no sentido da "sua capacidade de irrupção naquilo que se encontra bloqueado de criar, de seu teor de liberdade em se desfazer dos códigos que procuram explicar dando a tudo o mesmo sentido. O dispositivo tensiona, movimenta, desloca para outro lugar, provoca outros agenciamentos" (Benevides de Barros, 1997, p.189). A rede de contatos, como dispositivo, se põe a funcionar nos encontros, nos momentos de escuta, de trocas de experiências, de debates e da problematização dos desafios lançados pela PNH aos grupos de trabalho. Possibilita o entrelaçamento nos modos de trabalhar e produzir saúde e o estímulo às iniciativas e ao protagonismo das equipes, seguindo o princípio da inseparabilidade entre atenção e gestão, incide no enfrentamento da "fragmentação usual dos processos de trabalho" (Santos-Filho, Barros, 2007, p.207), apostando na indissociabilidade entre o planejar, o decidir, o executar e o avaliar.

Os processos de trabalho das equipes de saúde, tendo em vista os princípios da $\mathrm{PNH}$, instigam os grupos à revisão dos seus modos de operar. Isso equivale a dizer que as pessoas são capazes de desenvolver mudanças em si, no grupo e no ambiente (Campos, Amaral, 2007).

Entendemos a Rede enquanto um grupo, enquanto espaço de produção e criação (Barros, 2007). Podemos tomar esse campo grupal como um emaranhado de linhas e entrelaçamentos, que o compõem e formam redes, de identificações, de encontros e desencontros (Fernández, Del Cueto, 1985). Redes que produzem sentidos e sujeitos, construindo os caminhos e as histórias dos coletivos.

Quando iniciamos com a implementação da PNH no HCPA, no início de 2005, nos deparamos com as idéias de humanização voltadas para o senso comum, ou seja, aquelas atribuídas à boa educação ou à caridade dos sujeitos implicados nos processos de trabalho, na época consonantes com os pressupostos do então Programa Nacional de Humanização da Assistência Hospitalar (PNHAH), cujo objetivo era o de promover uma mudança de cultura no atendimento de saúde no Brasil, por meio da implementação de ações humanizadoras. 
O PNHAH propõe um conjunto de ações integradas que visam mudar substancialmente $\mathrm{O}$ padrão de assistência ao usuário nos hospitais públicos do Brasil, melhorando a qualidade e a eficácia dos serviços hoje prestados por estas instituições. É seu objetivo fundamental aprimorar as relações entre profissional de saúde e usuário, dos profissionais entre si, e do hospital com a comunidade. Ao valorizar a dimensão humana e subjetiva, presente em todo ato de assistência à saúde, o PNHAH aponta para uma requalificação dos hospitais públicos, que poderão tornar-se organizações mais modernas, dinâmicas e solidárias, em condições de atender às expectativas de seus gestores e da comunidade. (Brasil, 2001)

Nessa ocasião identificamos que, nas ações humanizadoras propostas por grupos e equipes, havia fragmentos possíveis de serem resgatados e ampliados à luz da $\mathrm{PNH}$ e seus dispositivos.

Cabe retomar que, até 2004, o Hospital atrelava a humanização a um Programa de Humanização até então existente, com iniciativas independentes, unidirecional, na maioria das vezes indicadas pelas chefias e sem caráter de continuidade.

A temática da humanização destacada pelo PNHAH e problematizada pela $\mathrm{PNH}$, posteriormente, já era tangenciada em outros espaços públicos de discussões sobre a saúde. Neste sentido destacamos as discussões e avanços possibilitados pela $8^{a}$ Conferência Nacional de Saúde.

O relatório final da $8^{a}$ Conferência Nacional de Saúde, de 1986, serviu de base à conquista da sociedade brasileira, organizada no Movimento da Reforma Sanitária, para que, na Constituição Federal de 1988, fosse reconhecido o direito à saúde como direito de todos e dever do Estado, estabelecendo a universalidade, a integralidade, a equidade, a descentralização, a regionalização e a participação da população como os princípios e diretrizes legais do Sistema Único de Saúde (SUS). As Leis Orgânicas da Saúde (n.8.080/90 e n.8.142/90) regulamentam esses princípios, reafirmando a saúde como direito universal e fundamental do ser humano.

O conceito ampliado de saúde, elaborado nessa Conferência, define que "saúde é a resultante das condições de alimentação, habitação, educação, renda, meio ambiente, trabalho, transporte, emprego, lazer, liberdade, acesso e posse da terra e acesso a serviços de saúde". Essa definição envolve reconhecer o ser humano como ser integral e a saúde como qualidade de vida. Passados 13 anos da criação do SUS, em 2003, o Ministério da Saúde (MS) decide priorizar o tema da humanização como aspecto fundamental a ser contemplado nas políticas de saúde.

A $11^{\text {a }}$ Conferência Nacional de Saúde inicia um processo mais amplo de reflexão, destacando a temática sobre a humanização, que posteriormente será priorizada em todo o conteúdo do Relatório Final da $12^{a}$ Conferência Nacional de Saúde, nos seus nove eixos temáticos, que dá ênfase à humanização dos serviços de saúde - sendo esta nas relações e nos atendimentos, na qualidade de vida no trabalho, no caráter multiprofissional do trabalho na saúde e na rejeição de qualquer tipo de preconceito. No eixo temático II, na diretriz 47 desse relatório, está prevista a implantação de uma política de humanização.

Esta política vem substituir o Programa Nacional de Humanização da Assistência Hospitalar (PNHAH) de 2000, que buscou implementar, no SUS, o conceito de atendimento humanizado.

No sentido desse resgate e ampliação foi que, alicerçado nos princípios da PNH, o GTH criou a Rede de Contatos. Esta Rede foi formada por um conjunto de setores ou locais de trabalho interessados na temática da humanização, os quais foram contatados pelos componentes do GTH com o objetivo de oportunizar a estas diferentes áreas o diagnóstico das fragilidades ou inconformidades nos processos de gestão e atenção, assim como identificar as necessidades em termos de humanização tanto do ambiente de trabalho para os profissionais quanto para os usuários. Com isso surgiram propostas e iniciativas voltadas à implementação de melhorias, favorecendo a construção de um ambiente, onde a política de humanização fosse a base das práticas e dos processos decisórios quanto à atenção em saúde.

A experiência das redes no Hospital de Clínicas vem se estruturando, desde que surgiu a ideia de uma interseção interna entre as iniciativas humanizadoras, efetivada por meio do contato realizado pelo GTH com as diferentes áreas e serviços do hospital, no sentido de sensibilizá-las e dar visibilidade institucional às iniciativas já existentes. Após vários encontros envolvendo a ouvidoria institucional, o Grupo de Trabalho Gestão do Cliente - com a análise dos resultados das pesquisas de satisfação - e de 
reuniões realizadas com diferentes grupos e serviços, delimitaram-se as seis primeiras áreas convidadas a comporem a rede. As áreas são aqui definidas como espaços, unidades ou serviços - que, no caso deste hospital, pode ser a recepção de um ambulatório, ou uma área administrativa ou financeira, ou, ainda, um Centro Cirúrgico ou uma Unidade de Cuidados Intensivos. Tais áreas foram se dispondo de modo a, juntas, comporem uma Rede, que a cada ano vem se ampliando, com a adesão de novas áreas e implementação de outras iniciativas humanizadoras.

Inicialmente, a Rede de Contatos foi constituída das seguintes áreas: Serviço de Processamento de Roupas, Serviço de Marcação e Coleta de Exames, Centro Obstétrico, Serviço de Emergência, o Morgue (local onde os familiares aguardam o preparo do corpo para efetuar o translado e funeral) e as Unidades de Internação de Pacientes, as quais foram identificadas pelas iniciativas de humanização já existentes, por serem apontadas tanto pela Ouvidoria como pela Pesquisa de satisfação do Cliente como espaços que necessitavam o trabalho do GTH ou por serem áreas de origem dos componentes do GTH.

No sentido de consolidar a Rede, o GTH reunia-se com as áreas e/ou suas parcerias, coordenando as atividades de integração, troca de experiências e fomento a novas práticas e modos de gestão e trabalho. As reuniões eram realizadas com os contatos das áreas (trabalhadores identificados com a temática da humanização e que se encarregaram, nos seus locais de trabalho, de fazerem a interface entre o GTH e as ações de humanização ali desenvolvidas), mas, também, com a presença dos gestores dessas áreas e demais trabalhadores. Acompanhavam-se in loco os processos de implementação dos dispositivos, realizavam-se apresentações públicas e geravam-se avaliações. Esses espaços/

acontecimentos estimulavam ao grupo em questão e inspiravam aos demais em busca de melhorias nos processos de trabalho. Aqui compreendemos estes espaços enquanto acontecimento no sentido de sua potência disrruptiva, do impacto e do rompimento que possibilitam (Carreteiro, 2003 p.268). A apresentação das ações, por parte das áreas, propiciava, neste sentido, ambiente para a reflexão das práticas, provocando a revisão das relações de trabalho e oportunizando iniciativas para avanços nos processos de atenção e gestão.

\section{Resultados e discussão}

O GTH lançou mão de diferentes estratégias e ações para a sensibilização quanto à temática da humanização e para a composição e ampliação da Rede de Contatos. Destacamos diferentes ações, tais como: revisão de processos de trabalho com impacto para os trabalhadores e usuários, salientando-se as melhorias no ambiente físico e psicológico; criação do Link - L-Humanização, que é uma forma de contato com o GTH por meio de acesso direto na página da intranet do HCPA, facilitando, agilizando e transversalizando a comunicação, realização de eventos com a temática da humanização, produções científicas e apresentação de pôsteres em encontros, jornadas e congressos. Outra ação a destacar, no sentido da propagação e contágio, é a publicação de textos no Jornal Espaço Aberto (jornal com circulação interna, produzido no HCPA), divulgando os dispositivos e as ações pró-humanização na instituição, bem como a produção da "carta de intenções" (iniciativa das diversas associações de profissionais, juntamente com a Administração Central do Hospital de Clínicas) para a otimização das relações interpessoais.

Durante o primeiro ano (2005-2006), as seis áreas da rede interagiram por meio de reuniões, encontros e debates promovidos pelo GTH, propagando suas ações e iniciativas e contagiando outras áreas. Estas áreas, inicialmente, desenvolveram estratégias para melhorar seus processos de trabalho, de atenção e gestão. Três delas concentraram-se no dispositivo ambiência, uma das áreas no acolhimento e as duas restantes no dispositivo saúde do trabalhador.

As Unidades de Internação Pediátrica desenvolveram melhorias após um diagnóstico realizado pelo projeto de pesquisa "Avaliação das ações humanizadoras desenvolvidas na Pediatria do Hospital de Clínicas de Porto Alegre" (Salgueiro et al., 2007), apresentando as melhorias produzidas por intermédio das intervenções com o grupo de pais.

A área da lavanderia canalizou as suas iniciativas para a valorização e o reconhecimento do trabalho desses profissionais, culminando na criação do dia do "Processador de Roupas". A terceira unidade foi 
o Centro Obstétrico, que iniciou seus processos com o "parto humanizado", ampliando seus indicadores a quase $100 \%$ de acompanhamento no momento do parto, com a presença do pai ou outro familiar, além de instituir as medidas não farmacológicas no momento do parto.

O Morgue foi um espaço que despertou interesse e discussão de várias áreas, a começar pelo Comitê de Ética. Reformas estruturais no ambiente, melhorias de processos (revisão da certidão de óbito), organização do ambiente - com o respeito às diferentes crenças e religiosidades - e melhor acolhimento aos familiares foram esforços conjuntos que transformaram o ambiente, suas salas, divisórias e seus corredores.

Nesse período, também houve a criação de espaços de discussão permanente por meio de Rodadas de conversa - método adaptado do "Método da Roda" (Campos, 2000) -, realizadas em diferentes áreas da instituição, com a participação multiprofissional. O envolvimento das áreas com a implantação de dispositivos da $\mathrm{PNH}$, encontros com demais apoiadores da $\mathrm{PNH}$, associações interprofissionais, atividades de extensão pró-humanização, encontros macrorregionais pró-humanização, representaram iniciativas marcantes envolvendo profissionais, acadêmicos e professores da instituição. A partir de então, foram se ampliando os contatos, e, com o decorrer do tempo, novas áreas passaram a compor a rede, sendo que, no segundo ano, esta era constituída por 23, e, no terceiro ano, por quarenta áreas.

As ações do GTH tiveram a função de ligar experiências e potencializar os grupos para o protagonismo, utilizando-se de um "modelo de rede que comporta assimetrias de saber e poder, e cuja transformação depende da construção de práticas de co-responsabilização na direção de um processo em prol da autonomia das pessoas" (Souza, Moreira, 2008, p.333). A experiência da humanização foi sendo propagada e vivenciada por diferentes atores e sujeitos, implicados no processo de produção da saúde, buscando-se, por meio dessas ações, transversalizar a rede, que foi se potencializando e multiplicando-se. Ampliando, assim, o "grau de abertura que garante às práticas de saúde a possibilidade de diferenciação ou invenção, a partir de uma tomada de decisão que faz dos vários atores sujeitos do processo de produção da realidade em que estão implicados" (Benevides, Passos, 2005b, p.393), dando forma a um cenário que abarque a pluralidade das modalidades de invenção por intermédio da experiência do trabalho.

Apontamos, como facilitadores para a implantação da Rede de Contatos, o apoio institucional recebido por parte da Administração Central do HCPA com relação à constituição do GTH e a abertura para as propostas encaminhadas por este. O histórico de protagonismo percorrido pelo hospital, no que diz respeito a ações de inclusão e escuta a pacientes e familiares, como, por exemplo, a permanência conjunta de pais e filhos na unidade de internação pediátrica - que antecede ao Estatuto da Criança e do Adolescente (ECA) e o comitê de defesa dos direitos da criança hospitalizada, que há muito já foram implantados no cotidiano do hospital - foi um aspecto que contribuiu no sentido da aceitação das propostas da Política, bem como o incentivo e a concessão para que integrantes do GTH realizassem atualização quanto aos pressupostos teóricos da $\mathrm{PNH}$, por intermédio da participação em cursos promovidos pelo Ministério da Saúde.

Também ressaltamos aqui alguns aspectos que, em muitos momentos, se colocaram como obstáculos. Podemos destacar: o acúmulo e sobreposição de atividades tanto por parte dos integrantes do GTH como dos contatos da rede; a dificuldade em identificar as pessoas que fossem sensíveis e receptivas com a proposta da $\mathrm{PNH}$ e que, assim sendo, tivessem possibilidade de desenvolver ações no sentido da propagação dos seus pressupostos. E somado a isso, o desafio de implantar uma política que se diz transversal no núcleo de uma organização hierárquica. Fato que nos colocou, muitas vezes, na situação de buscar legitimação de ações e iniciativas desenvolvidas por protagonistas, muitas vezes com um lugar institucional de pouco reconhecimento e visibilidade. Dessa forma tornou-se fundamental a inclusão da escala hierárquica em toda e qualquer negociação, que envolvesse os processos de trabalho e as ações de saúde.

Esse cenário configurou o contágio institucional em relação aos princípios da PNH e seus dispositivos, na medida em que, nos encontros da Rede de Contatos, ia se dando visibilidade às experiências e iniciativas pró-humanização e transversalizando de modo a serem replicadas e reinventadas nos/e pelos diferentes grupos de trabalho. 


\section{Considerações finais}

O modelo de gestão, na grande maioria dos estabelecimentos de saúde, possui, como característica fundamental, a centralização de poder e pouco espaço para as ações democráticas. Em vários hospitais, os processos de gestão estruturam-se de forma quase inflexível, "fortalecendo os acordos internos entre grupos e promovendo uma política de favorecimentos e negociações veladas" (Abrahão, 2008. p.100).

Um sistema de poder verticalizado, com muitos níveis hierárquicos, em que as tomadas de decisões são centralizadas, induz ao descompromisso e à alienação entre a maioria dos trabalhadores. Um processo de trabalho centrado em procedimentos pode levar ao descomprometimento das equipes de saúde, reduzindo, assim, a corresponsabilidade nas ações e nos resultados.

Outra condição da qual não se pode alienar é que, na atualidade, o hospital ainda cultiva e mantém sua hegemonia sobre as ações de saúde, e assim reproduz um modelo de gestão pouco democrático, que reitera práticas de trabalho cristalizadas, que não favorecem a emergência de sujeitos transformadores dos modos de gerenciar e fazer saúde (Abrahão, 2008).

Nesta perspectiva, reforça-se a noção de integralidade e relações múltiplas interativas, onde se faz imprescindível a indissociabilidade do cuidado e do gerenciamento. Essa indissociabilidade provoca alterações nos modos de atenção e de gestão, culminando num alto grau de autonomia com aumento da responsabilidade dos trabalhadores, usuários e gestores (Pasche, 2007). Nesse compasso, "as equipes se tornam mais potencializadas quando têm reconhecido seu espaço social de atuação, com mais autonomia, crescimento nos conhecimentos técnicos e diálogo mais autêntico entre disciplinas" (Erdmann et al., 2006, p.486).

Embora o cotidiano do hospital submeta, constantemente, os profissionais a situações críticas e indesejáveis, como falta de leitos numa emergência super lotada e a escassez de recursos humanos provocadores de dilemas éticos, acreditamos que é possível a inter-relação entre todas as pessoas, sejam elas profissionais ou pacientes. Essa convivência propicia viver em harmonia, mesmo diante das tensões dos momentos mais difíceis, pois acreditamos que é possível trilharmos caminhos onde possamos demonstrar, com intensidade, a complexidade exigida pelo cuidado, bem como sua ambiguidade e implicações subjetivas enquanto ansiedade e preocupação, mas também atenção, desvelo e solicitude (Eizirik, 2002), e a consequente e preconizada humanização. É desta forma que o GTH vem estimulando a todos, por meio da construção da rede de contatos, a exercer essa prática aliada a constante reflexão e revisão dos modos de produzir saúde.

A configuração desta rede possibilita que as ações de humanização, permeando diferentes espaços e atores da organização, possam dar um fluxo que seja transversal aos interstícios dos organogramas e hierarquias organizacionais e das relações.

Tendo em vista a vivência das redes e o protagonismo dos sujeitos e atores em saúde, se faz necessário encontrar novas estratégias que propiciem soluções para a grave questão existente nas instituições de saúde, ou seja, para a massificação do atendimento ao ser humano. Mesmo todas as iniciativas postas em prática não têm sido suficientes.

Enfim, a humanização no universo contextualizado anteriormente decorre do olhar de compreensão e do protagonismo e alteridade dos profissionais de saúde. 


\section{Colaboradores}

As autoras contribuíram igualmente em todas as etapas do manuscrito.

\section{Referências}

ABRAHÃO, A.L. Colegiado gestor: uma análise das possibilidades de autogestão em um hospital público. Cienc. Saude Colet., v.13, n.1, p.95-102, 2008.

BACKES, D.S.; LUNARDI FILHO, W.D.; LUNARDI, V.L. O processo de humanização do ambiente hospitalar centrado no trabalhador. Rev. Esc. Enferm. USP, v.40, n.2, p.221-7, 2006.

BARROS, R.B. Grupo: a afirmação de um simulacro. Porto Alegre: Sulina/Editora da UFRGS, 2007.

BENEVIDES DE BARROS, R. Dispositivos em ação: o grupo. In: LANCETTI, A. (Org.). Saúde e loucura, subjetividade: questões contemporâneas. v.6. São Paulo: Hucitec, 1997. p.183-91.

BENEVIDES, R.B. A Psicologia e o Sistema Único de Saúde: quais interfaces? Psicol. Soc., v.17, n.2, p.21-5, 2005.

BENEVIDES, R.; PASSOS, E. A humanização como dimensão pública das políticas de saúde. Cienc. Saude Colet., v.10, n.3, p.561-71, $2005 a$.

Humanização na saúde: um novo modismo. Interface - Comunic., Saúde, Educ., v.9, n.17, p.389-406, 2005b.

BRASIL. Ministério da Saúde. Secretaria de Atenção à Saúde. Núcleo Técnico da Política Nacional de Humanização. HumanizaSUs: documento base para gestores e trabalhadores do SUS. Brasília: Ministério da Saúde, 2006.

Ministério da Saúde. Secretaria de Atenção à Saúde. Programa Nacional de

Humanização da Assistência Hospitalar. Brasília: Ministério da Saúde, 2001.

Disponível em: <http://bvsms.saude.gov.br/bvs/publicacoes/pnhah01.pdf > . Acesso em: 23 jun. 2009.

CAMPOS, G.W.S. Um método para a análise e co-gestão de coletivos: a construção do sujeito, a produção de valor de uso e a democracia em instituições - o método da roda. São Paulo: Hucitec, 2000.

CAMPOS, G.W.S.; AMARAL, M.A. A clínica ampliada e compartilhada, a gestão democrática e redes de atenção como referenciais teórico-operacionais para a reforma do hospital. Cienc. Saude Colet., v.12, n.4, p.849-59, 2007.

CARRETEIRO, T.C. Acontecimento: categoria biográfica individual, familiar, social e histórica. In: FÉRES-CARNEIRO, T. (Org.). Família e casal: arranjos e demandas contemporâneas. Rio de Janeiro: Ed. PUC-Rio/ Loyola, 2003. p.267-85.

EIZIRIK, M.F. Michel Foucault: um pensador do presente. Ijuí: Ed. Unijuí, 2002.

ERDMANN, A.L. et al. Gestão das práticas de saúde na perspectiva do cuidado complexo. Texto Contexto Enferm., v.15, n.3, p.483-91, 2006.

FERNÁNDEZ, A.M.; DEL CUETO, A.M. El dispositivo grupal. In: PAVLOVSKY, E. (Org.). Lo grupal 2. Buenos Aires: Ediciones Busqueda, 1985. p.13-56. v.2.

KAMKHAGI, V.R. Horizontalidade, verticalidade e transversalidade em grupos. In: BAREMBLITT, G. (Org.). Grupos: teoria e técnica. 2.ed. Rio de Janeiro: Graal, 1986. p.205-19.

PASCHE, D.F. A reforma necessária do SUS: inovações para a sustentabilidade da política pública de saúde. Cienc. Saude Colet., v.12, n.2. p.312-4, 2007. 
RIGHI, L.B. Produção de redes de atenção à saúde: acordos, confrontos e reparos. In: PASCHE, D.; CRUZ, I. (Orgs.). A Saúde Coletiva: diálogos contemporâneos. Ijuí: Ed.Unijuí, 2006. p.73-92.

SALGUEIRO, J.B. et al. Avaliação das ações humanizadoras desenvolvidas na pediatria do Hospital de Clínicas de Porto Alegre. Rev. HCPA, v.27, n.2, p.5-9, 2007.

SANTOS FILHO, S.B. Perspectivas da avaliação na Política Nacional de Humanização em Saúde - PNHS: aspectos conceituais e metodológicos. Cienc. Saude Colet., v.12, n.4, p.999-1010, 2007.

SANTOS-FILHO, S.B.; BARROS, R.B. Câmara técnica de humanização como dispositivo de co-gestão: experimentando o conceito de Rede. In: SANTOS-FILHO, S.B.; BARROS M.E.B. (Orgs.). Trabalhador da Saúde: muito prazer! Protagonismo dos trabalhadores na gestão do trabalho em saúde. Coleção Saúde Coletiva. Ijuí: Ed.Unijuí, 2007. p.20332.

SOUZA, W.S.; MOREIRA, M.C.N. A temática da humanização na saúde: alguns apontamentos para debate. Interface - Comunic., Saude, Educ., v.12, n.25, p.327-38, 2008 . 
Este estudo enfoca a rede de contatos como estratégia metodológica da Política Nacional de Humanização (PNH). Trata-se de um relato de experiência, instituído no Hospital de Clínicas de Porto Alegre (HCPA), que buscou identificar as ações de humanização suscitadas pela formação da Rede de Contatos no âmbito da instituição. O Grupo de Trabalho de Humanização do HCPA procurou estruturar uma Rede que abrangesse o maior número possível de áreas no hospital, iniciando um trabalho de sensibilização dentro dos setores e buscando promover ações convergentes com as propostas pela PNH. Observou-se que, desde 2005 até agora, várias ações humanizadoras aconteceram nas diversas áreas envolvidas, demonstrando que o dispositivo das redes é ferramental precioso para a instituição, pois oferece indicadores para a $\mathrm{PNH}$ e favorece a interrelação entre os diferentes atores que buscam, para além das dificuldades de um cotidiano hospitalar, a promoção de outros modos de fazer em saúde.

Palavras-chave: Humanização da assistência. Política de saúde. Redes comunitárias. Assistência hospitalar.

The network as a methodological strategy for the National Humanization Policy: the experience of a university hospital

This study focuses on contact networks as methodological strategies for the National Humanization Policy (PNH). It reports on experience instituted at Hospital de Clínicas de Porto Alegre (HCPA), in which it was sought to identify humanization actions that arose through the formation of contact networks within the scope of the institution. HCPA's Humanization Work Group sought to structure a network that would encompass the largest possible number of sectors in the hospital, thus starting awareness-raising work within these sectors and seeking to promote actions convergent with the $\mathrm{PNH}$ proposals. It was observed that, from 2005 to today, several humanizing actions occurred in the various sectors involved, thereby demonstrating that the network device is a precious tool for the institution, since it provides indicators for PNH and favors interrelationships between the different players that seek to go beyond the difficulties of everyday hospital activity, to promote other means of healthcare practices.

Keywords: Humanization of care. Healthcare policy. Community networks. Hospital care.

\section{Da red como estrategia metodológica de la Política Nacional de Humanización: la experiencia en un hospital universitario}

Este estudio enfoca la red de contactos como estrategia metodológica de la Política Nacional de Humanización (PNH). Se trata de un relato de experiencia instituido en el Hospital de Clínicas de Porto Alegre (HCFA), estado de Rio Grande do Sul, Brasil, que trató de identificar las acciones de humanización suscitadas por la formación de la Red de Contactos en el ámbito de la institución. El Grupo de Trabajo de Humanización del HCPA procuró estructurar una Red que comprendiese el mayor número posible de áreas en el hospital, iniciando un trabajo de sensibilización dentro de los sectores y tratando de promover acciones convergentes con las propuestas por la $\mathrm{PNH}$. Se ha observado que, desde 2005 hasta ahora, varias acciones humanizadoras se producen en las diferentes áreas, demostrando que el dispositivo de las redes es instrumento precioso para la institución pues ofrece indicadores para la PNH y favorece la inter-relación entre los diferentes actores que procuran, más allá de las dificultades de un cotidiano hospitalario, la promoción de otros modos de hacer en salud.

Palabras clave: Humanización de asistencia. Política de salud. Redes comunitarias. Asistencia hospitalaria. 